\title{
A dynamically polarized target for CLAS12
}

\section{C.D. Keith*†}

Thomas Jefferson National Acccelerator Facility

E-mail: ckeith@jlab.org

The $12 \mathrm{GeV}$ physics program in Hall B at Jefferson Lab will be centered on the newly commissioned, high acceptance spectrometer CLAS12 and will utilize a variety of nuclear targets, including a dynamically polarized solid target for experiments on longitudinally polarized protons, deuterons, and lithium nuclei. The target, under construction in a collaborative effort between the Jefferson Lab Target Group, Christopher Newport University, Old Dominion University, and the University of Virginia, is designed with a number of innovative features. These include multiple target cells for reduced systematic uncertainties, internal superconducting shim coils to adjust the polarizing magnetic field, and a $1 \mathrm{~K}$ evaporation refrigerator with an integrated load lock system for the target samples. The current status of the system's design, construction, and various R\&D efforts are described.

XVII International Workshop on Polarized Sources, Targets \& Polarimetry

16-20 October 2017

Kaist, South Korea

\footnotetext{
*Speaker.

${ }^{\dagger}$ On behalf of the Jefferson Lab Target Group and the CLAS collaboration
} 


\section{Introduction}

At the time of this workshop, Jefferson Lab has recently completed its $12 \mathrm{GeV}$ project, a three-year-long, \$338M effort to double the energy of the CEBAF accelerator. The project has also added a fourth experimental end station at JLab, Hall D, and upgraded the spectrometer systems of two of the original three end stations, Halls B and C. Just as polarized solid targets were an integral part of the lab's physics program during its $6 \mathrm{GeV}$ era [1], they seem likely to play an even larger role in its future. Nowhere is this more true than in Hall B, where three such targets were used inside its $4 \pi$ spectrometer, CLAS [2]: a $1 \mathrm{~K}, 5 \mathrm{~T}$ dynamically-polarized target of $\mathrm{NH}_{3}$ and $\mathrm{ND}_{3}$ [3], a dynamically-polarized, frozen spin target of butanol [4], and a brute-force polarized, frozen spin target of solid HD [5]. For physics at $12 \mathrm{GeV}$, CLAS has been completely replaced by an even larger detector package, CLAS12, which like its predecessor, was explicitly designed with polarized targets in mind. In fact, solid polarized targets will be utilized for approximately one-third of the beam time thus far approved, or conditionally approved in Hall B. The bulk of that time will feature a new target with longitudinal spin direction and dynamically polarized at $5 \mathrm{~T}$ and $1 \mathrm{~K}$. This target is currently under construction by a collaboration between JLab, Christopher Newport University, Old Dominion University, and the University of Virginia.

Like its predecessor [3], the new target system will be used to mostly polarize samples of preirradiated $\mathrm{NH}_{3}$ and $\mathrm{ND}_{3}$ (but also ${ }^{6} \mathrm{LiH}$ and ${ }^{7} \mathrm{LiD}$ ), but it will differ dramatically in other aspects:

- The previous system [3] utilized a vertically-oriented ladder which permitted rapid changes between multiple target samples. This will not be possible in the new system because the tighter geometrical constraints of CLAS12 impose a purely horizontal geometry;

- Unlike the dedicated superconducting magnet used for the $6 \mathrm{GeV}$ target, the magnet systems of CLAS12 will provide the $5 \mathrm{~T}$ polarizing field for the new target;

- The new system is expected to operate with luminosities an order-of-magnitude higher than the previous one, with proton luminosities up to $10^{35} \mathrm{~cm}^{-2} \mathrm{~s}^{-1}$;

- For the new target, we will attempt to simultaneously polarize two samples, in opposite spin directions, rather than alternate the direction of a single sample sequentially.

The remainder of this article will focus on the magnet and refrigeration systems for the new target. Investigations of a new NMR system for measuring the sample polarizations are also underway and are the subject of a separate proceedings from this Workshop [6].

\section{Magnet systems}

\subsection{Polarizing magnet}

As was the case for the $6 \mathrm{GeV}$ spectrometer CLAS, a superconducting torus magnet is used to track charged particles for the detector packages of CLAS12. However, CLAS12 also features a $5 \mathrm{~T}$ superconducting solenoid located upstream of the torus (Fig. 1). This magnet has three main purposes. First, its field provides tracking for the so-called Central Detector System, composed of a Silicon Vertex Tracker (SVT), Central Time of Flight system (CTOF), Micromegas Vertex Tracker (MM), and the Central Neutron Detector (CND). Second, the solenoid focuses Møller-scattered 


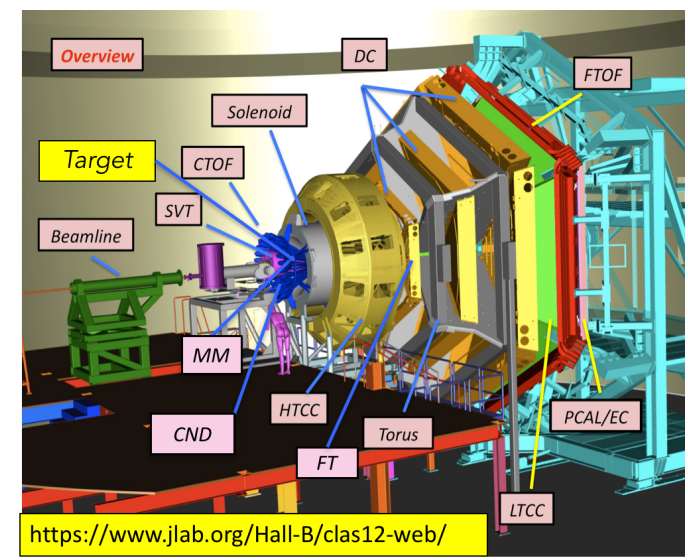

Figure 1: Overview of the CLAS12 spectrometer system. Additional details may be found at the link indicated.

electrons away from the downstream detector system. Third, it generates a highly uniform, $5 \mathrm{~T}$ field for dynamically polarized targets.

The solenoid, shown in Fig. 2, features five superconducting coils wound in series. The outermost coil, number 5 , is wound in the opposite sense from the other four to reduce the fringe field outside the magnet's $0.8 \mathrm{~m}$ diameter warm bore. For optimum polarization of protons and deuterons using dynamic nuclear polarization (DNP), the magnet was specified to provide a uniformity of $\Delta B / B \leq 10^{-4}$ over a cylindrical target volume $\varnothing 20 \times 40 \mathrm{~mm}^{2}$. A preliminary field map performed at JLab indicates a uniformity about three times the specified value. Based on polarization versus field curves of both $\mathrm{NH}_{3}$ and $\mathrm{ND}_{3}$ [7], we believe the impact on the proton polarization will be small, but the deuteron polarization could suffer $10-20 \%$. To address this, we plan to incorporate small shim coils inside the target cryostat to improve the field uniformity. These shims can also be utilized to polarize smaller, $20 \mathrm{~mm}$ long samples in opposing directions.
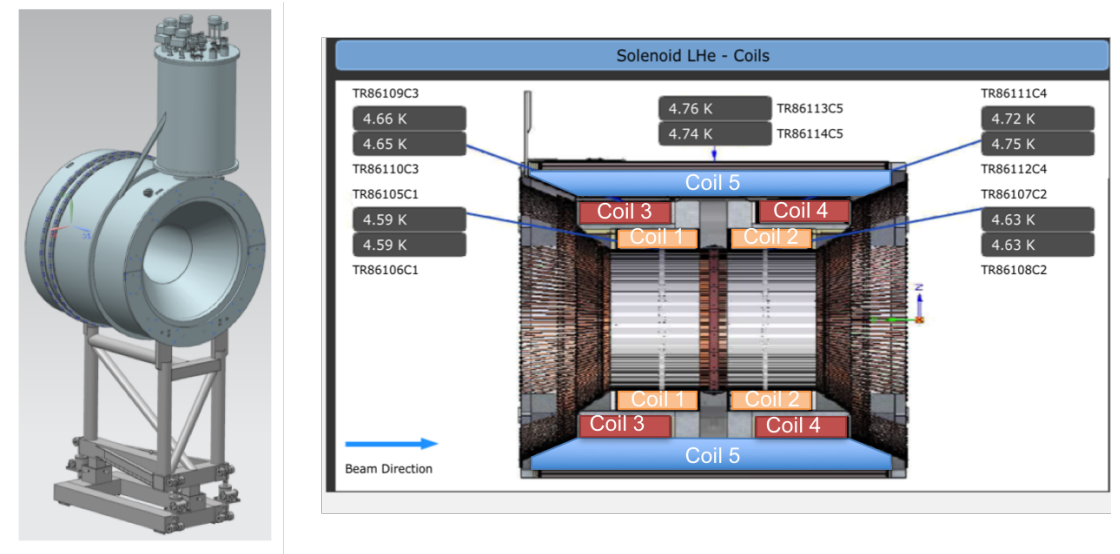

Figure 2: 3D and diagrammatic drawings of the CLAS12 solenoid. A field of $5 \mathrm{~T}$ is generated at $2400 \mathrm{~A}$. 


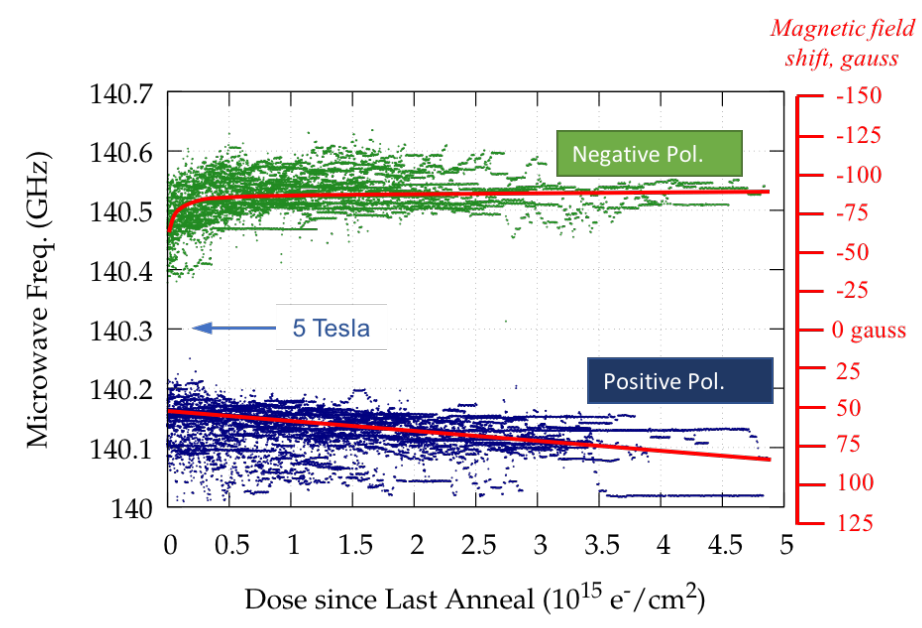

Figure 3: Optimum microwave frequencies for polarizing both spin states of $\mathrm{NH}_{3}$ at $5 \mathrm{~T}$ as a function of accumulated electron dose [9]. The corresponding magnetic field shifts from $5 \mathrm{~T}$ are indicated on the left. The red lines are guides to the eye.

\subsection{Shim coils for dual targets}

A single target sample is utilized in the vast majority of polarized target scattering experiments, and its spin direction is periodically reversed so that data on the two polarization states is accumulated sequentially. In the case of dynamically polarized targets, the reversal can be performed by either adjusting the frequency of the microwaves used to drive the DNP process, or less commonly, fixing this frequency and adjusting the static magnetic field. However, it is possible to use two samples, polarized in opposite directions, and acquire data on the two polarization states simultaneously. Despite some technical difficulties, this has the potential to reduce certain systematic errors, such as those arising from drifts in detector efficiencies.

Dual polarized target cells were first implemented by the Spin Muon Collaboration (SMC) more than two decades ago [8]. The SMC collaboration (and later the COMPASS collaboration) dynamically polarized the two samples in a uniform $2.5 \mathrm{~T}$ field utilizing different microwave frequencies. A "stopper", comprising copper and graphite disks, was located between the cells to isolate each from the wrong microwave source. For the present target system, we intend to try the alternative method: one microwave frequency and two magnetic fields. The shim coil system previously mentioned to improve the uniformity of the CLAS12 solenoid will also be able to increase the field in one $\varnothing 20 \times 20 \mathrm{~mm}^{2}$ sample volume, and decrease it in another. With the microwave source tuned halfway between the normal frequencies for positive and negative proton polarization, the sample in the higher field will be polarized positively, and the one in the lower field negatively. We feel this method has two possible advantages. First, it obviates the need for a microwave stopper, thus reducing the amount of unwanted material in the beam path. Second, because the samples will have different Larmor frequencies, there is no potential for crosstalk between the two NMR systems used to measure the polarization.

As shown in Fig. 3, the microwave frequencies for positive and negative polarization in freshly- 


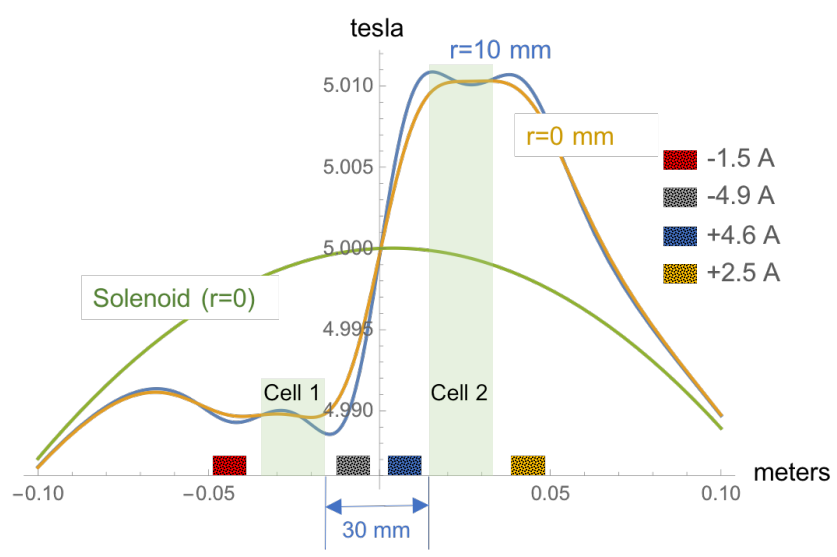

Figure 4: Shim coil simulation for a dual-cell target with $30 \mathrm{~mm}$ separation. A polynomial fit to the solenoid field map (on-axis) is shown in green, while the net field with shims (on-axis and $10 \mathrm{~mm}$ off-axis) is plotted in orange and blue. The position of the two target cells are indicated by the green bars, while the approximate coil locations are shown by the small, colored boxes. The coil currents, indicated on the right, adjust the net field around the target cells by approximately $\pm 100 \mathrm{G}$.

irradiated $\mathrm{NH}_{3}$ are separated by approximately $0.3 \mathrm{MHz}$ at $5 \mathrm{~T}$. The separation increases to about $0.4 \mathrm{MHz}$ as the ESR line of paramagnetic radicals in the sample broadens with accumulated electron dose. Alternatively, the magnetic field difference required to polarize the two spin states starts at approximately $120 \mathrm{G}$ and increases to $160 \mathrm{G}$.

The shims will consist of four separate coils installed inside the target cryostat and cooled to about $1 \mathrm{~K}$. Initial studies indicate that the required fields can be generated by a few layers of $\varnothing 0.18 \mathrm{~mm} \mathrm{NbTi}: \mathrm{Cu}$ wire at currents less than $5 \mathrm{~A}$. The results of one design study, with a $30 \mathrm{~mm}$ separation between the target samples, is shown in Fig. 4. Each coil consists of three layers of 55 turns, wound on an inner diameter of $54 \mathrm{~mm}$. Alternatively, the two outermost coils can both be energized with positive current $(\sim 0.5 \mathrm{~A})$, while the inner two are unused. This configuration would improve the solenoid uniformity to better than $10^{-4}$ and may be an attractive option for polarizing a single, $40 \mathrm{~mm}$ long $\mathrm{ND}_{3}$ sample.

\section{Refrigerator}

The target samples will be cooled to approximately $1 \mathrm{~K}$ by a horizontal, Roubeau-style, ${ }^{4} \mathrm{He}$ evaporation refrigerator [10]. Based on a total $\mathrm{NH}_{3}$ mass of about $8 \mathrm{~g}$ and an electron beam current of $10 \mathrm{nA}$, we expect the heat loads to be approximately $160 \mathrm{~mW}$ from microwaves and $50 \mathrm{~mW}$ from the beam. To allow sufficient headroom for additional heat sources, the refrigerator is designed to provide a cooling power of approximately $0.8 \mathrm{~W}$ at $1 \mathrm{~K}$.

Liquid helium at $4.2 \mathrm{~K}$ and $1 \mathrm{~atm}$ will be provided from a 5001 dewar, which in turn is continuously replinished from JLab's End Station Refrigeration facility. Upon entering the target cryostat, the helium first passes a sintered metal plate to separate the vapor from the liquid. The vapor is pumped from this separator with a small membrane pump and used to cool a set of upstream baffles as well as a copper heat shield surrounding the downstream portions of the refrigerator. Liquid 


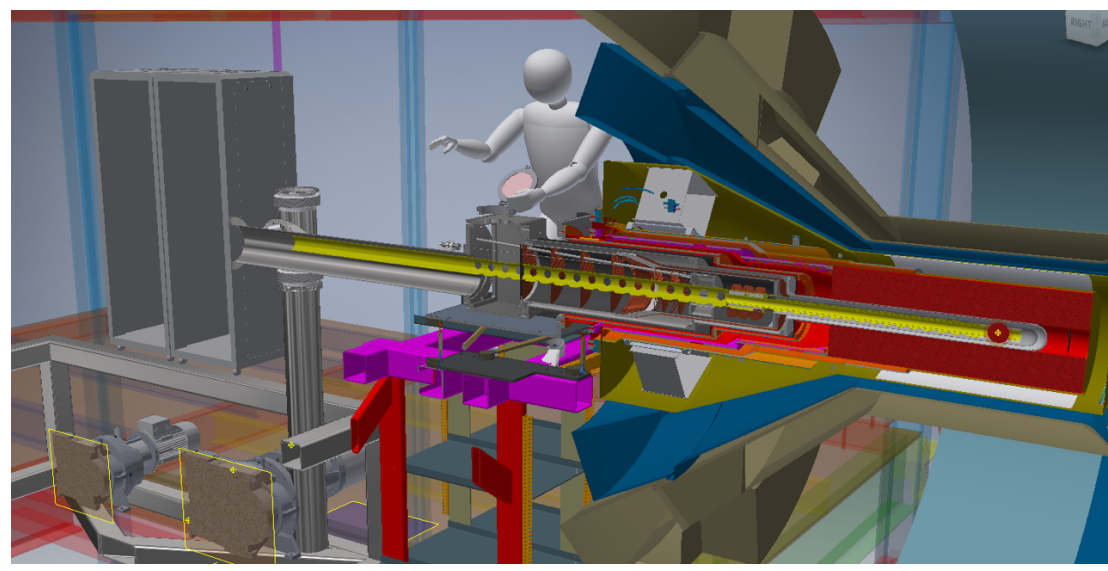

Figure 5: 3D model of the longitudinally polarized target inside the Central Detector System of CLAS12. Samples of frozen $\mathrm{NH}_{3}$ and $\mathrm{ND}_{3}$ are loaded into the via a load-lock mechanism on the upstream portion of the cryostat.

drained from the separator flows through a heat exchanger where it transfers heat to the cold vapor pumped from the refrigerator at $1 \mathrm{~K}$. The subcooled liquid then expands across a miniature JouleThompson valve and fills a small bath surrounding the target samples. Gas evaporating from this bath is pumped across the heat exchanger by a set of roots and rotary-vane pumps with a measured speed of $5400 \mathrm{~m}^{3} / \mathrm{h}$ at 0.1 torr.

\section{Target samples}

The target samples will consist of mm-sized frozen granules of ammonia $\left(\mathrm{NH}_{3}\right.$ or $\left.\mathrm{ND}_{3}\right)$ loaded into perforated PCTFE containers with aluminum windows for the beam. These containers will be filled prior to the experiment and stored under $\mathrm{LN}_{2}$ until needed. To put them into the beam, the aforementioned LHe bath will be retracted to the upstream end of the cryostat, and the containers placed into it via a load-lock port on the cryostat (Fig. 5). The bath is then returned to the downstream end of the refrigerator where it is filled with $1 \mathrm{~K}$ liquid before dynamic polarization is commenced.

After the samples accumulate an electron dose of approximately $5 \times 10^{15} \mathrm{~cm}^{-2}(2-3$ days at $10 \mathrm{nA}$ ), the radiation damage they have incurred must be repaired by warming them to about $90 \mathrm{~K}$ for several minutes. This annealing process is normally accomplished in situ by heating the samples with warm helium gas. In our experience at JLab, it can be difficult to warm the samples to a uniform, well-determined temperature using this technique. For the target system described here, we will take a different approach and simply replace the spent target samples with fresh ones. After they are removed from the beam, the radiation-damaged samples will be annealed in a bath of liquid argon before they are returned to service. The temperature of this bath can be easily adjusted by varying its saturated vapor pressure. 


\section{Summary}

We have presented the preliminary design of a new dynamically polarized solid target, currently under construction at Jefferson Lab. It is designed to include a number of features never before utilized at JLab, including dual-cell targets and an innovative load-lock mechanism for placing the cells in the beam. The target will be at the foundation of a new longitudinal spin physics program with the CLAS12 spectrometer in Hall B and is intended to provide a proton luminosity of $10^{35} \mathrm{~cm}^{-2} \mathrm{~s}^{-1}$ at an incident beam current of $10 \mathrm{nA}$.

\section{Acknowledgements}

This material is based upon work supported by the U.S. Department of Energy, Office of Science, Office of Nuclear Physics under contract DE-AC05-06OR23177.

\section{References}

[1] C.D. Keith et al., in Proceedings of PSTP 2015, PoS (PSTP2015) 013.

[2] B.A. Mecking et al., Nucl. Instr. and Meth. A 503 (2003) 513.

[3] C.D. Keith et al., Nucl. Instr. and Meth. A 501 (2003) 327.

[4] C.D. Keith et al., Nucl. Instr. and Meth. A 684 (2010) 27.

[5] M.M. Lowry et al., Nucl. Instr. and Meth. A 8154 (2016) 31.

[6] J.D. Maxwell, these proceedings.

[7] M.L. Seely, PhD thesis, Yale University (1982).

[8] D. Adams et al., Nucl. Instr. and Meth. A 437 (1999) 23.

[9] J.D. Maxwell et al., Nucl. Instr. and Meth. A 885 (2018) 145.

[10] P. Roubeau, Cryogenics 6 (1966) 207. 